\title{
Infected Urachal Cyst Following Laparoscopic Cholecystectomy
}

\author{
Max Jackson, BA, Antonio Cusano, BS, Gregory Murphy, MD, Peter Haddock, PhD, \\ Anoop Meraney, MD, Joseph Wagner, MD \\ Urology Division, Hartford Healthcare Medical Group, Harfford, Connecticut (all authors).
}

\begin{abstract}
Introduction: In an era of ultrasonography and computed tomography (CT), urachal remnants have been detected with increasing frequency. If these remnants become infected, they can mimic a variety of intra-abdominal pathologies. We present the case histories of two patients with an infected urachal cyst that developed after laparoscopic cholecystectomy and necessitated excision.

Case Descriptions: Patient 1: Four years after a laparoscopic cholecystectomy, a 36-year-old man presented with dysuria, abdominal pain, leukocytosis, and fluid leakage from the umbilical port site. CT imaging revealed an infected urachal cyst with an adherent loop of sigmoid colon. Antibiotic treatment preceded laparoscopic excision of the urachal cyst with partial cystectomy and closure of the sigmoid-to-urachus fistula. In a 3-year follow-up, there was no recurrence. Patient 2: A 68-year-old woman presented 11 months after laparoscopic cholecystectomy with abdominal pain, intermittent fever, and leukocytosis. CT imaging revealed an infected urachal cyst with an associated phlegmon in the abdominal wall. Antibiotic treatment preceded two incision-and-drainage procedures. Six weeks later, the patient underwent robotic excision of the urachal cyst and partial cystectomy. A 3-year follow-up showed no recurrence.

Discussion: The urachus can be punctured during laparoscopic periumbilical port placement and convert into a draining sinus or abscess. Subsequent infection can present with umbilical drainage, abdominal pain, urinary symptoms, and systemic infection. Surgical excision is a reasonable option once the acute infection has been treated. Any images that include the urachus should be reviewed before procedures involving an umbilical port, as puncture of urachal cysts may increase the risk of infection.
\end{abstract}

Key Words: Cholecystectomy, Infection, Laparoscopy, Urachal cyst.

Citation Jackson M, Cusano A, Murphy G, Haddock P, Meraney A, Wagner J. Infected urachal cyst following laparoscopic cholecystectomy. CRSLS e2014.00228. DOI: 10.4293/CRSLS.2014.00228.

Copyright $\odot 2015$ by SLS, Society of Laparoendoscopic Surgeons. This is an open-access article distributed under the terms of the Creative Commons Attribution-Noncommercial-ShareAlike 3.0 Unported license, which permits unrestricted noncommercial use, distribution, and reproduction in any medium, provided the original author and source are credited.

The authors declare no conflicts of interest.

Address correspondence to: Peter Haddock, PhD, Suite 416, 4th Floor, 85 Seymour Street, Hartford, CT 06106, USA. Telephone: 860-287-9208; Fax: 806-524-8643; E-mail: peter.haddock@hhchealth.org

\section{INTRODUCTION}

During the first 10 weeks of fetal development, urine drains from the bladder to the umbilicus through the urachus. Subsequently, during the 12th week of development, the urachus is obliterated, giving rise to a fibrous cord between the bladder and umbilicus called the median umbilical ligament. This process corresponds with the development of the urethra for bladder drainage. ${ }^{1,2}$

Urachal abnormalities, caused by aberrant sealing of the urachus during fetal development, ${ }^{3}$ can present as one of four subtypes: patent urachus, urachal sinus, urachal cyst, and urachal diverticulum. ${ }^{1,2,4}$ Before the routine use of modern imaging techniques, the prevalence of urachal cyst was unclear because it is usually asymptomatic and is found incidentally on abdominal ultrasonography or computed tomographic (CT) scan. ${ }^{2}$ However, in a study of children who underwent ultrasonography, urachal remnants were present in 99\% of cases. ${ }^{5}$ Furthermore, in another study, the persistence of urachal remnants was reported in $61.7 \%$ of patients $<16$ years, $49.0 \%$ of those 16 to 35 years, $20.4 \%$ of those 36 to 55 years, and $3.7 \%$ of 
Infected Urachal Cyst Following Laparoscopic Cholecystectomy, Jackson M et al.

those $\geq 56$ years of age. ${ }^{6}$ These findings concur with those in an autopsy study in which urachal remnants were found on microscopic examination in $32 \%$ of cases. ${ }^{7}$ The data support the suggestion that the urachus is a regressive structure that undergoes involution during a normal lifespan and is often present in normal adults. ${ }^{6}$

If a urachal cyst is ruptured during port placement in laparoscopic surgery, an abscess or a persistently draining sinus may develop that can be difficult to eradicate. Affected patients may present with fluid leakage from the umbilical port site; abdominal pain; urinary symptoms, such as hematuria or dysuria; and signs of systemic infection. ${ }^{2,8-12}$ CT imaging is usually necessary for diagnosis and surgical planning. ${ }^{13}$ Incision and drainage of the infected cyst may temporarily resolve the acute infection, but surgical excision is usually necessary to prevent recurrence. ${ }^{14,15}$ The procedure can be performed with laparoscopic or robotic surgical techniques. ${ }^{14-17}$

We report two cases of patients with a urachal cyst that became infected after laparoscopic cholecystectomy and was treated definitively with robotic excision including partial cystectomy. Neither patient had a recurrence of the urachal infection during a 3-year follow-up.

\section{CASE DESCRIPTIONS}

\section{Patient 1}

Immediately after undergoing laparoscopic cholecystectomy, a 36-year-old, morbidly obese man reported the leakage of clear fluid from the site of the periumbilical port. The drainage was intermittent for 4 years before presentation, but no treatment was initiated. In addition, the patient reported dysuria and increasing abdominal pain for 6 to 12 months before the consultation. He presented acutely with signs of systemic infection, including low-grade fevers, tachycardia, and leukocytosis. Subsequent CT imaging revealed an infected urachal cyst with a suspected fistula to the adjacent area of the sigmoid colon (Figure 1). A robot-assisted laparoscopic excision of the urachal cyst was performed successfully, in addition to a partial cystectomy and closure of the sigmoid colon-tourachus fistula. Pathologic analysis revealed inflammatory changes, but no evidence of malignancy. The patient had no recurrence of urachal infection in a 3-year follow-up.

\section{Patient 2}

A 68-year-old woman underwent a laparoscopic cholecystectomy for acute cholecystitis. She presented to our clinic

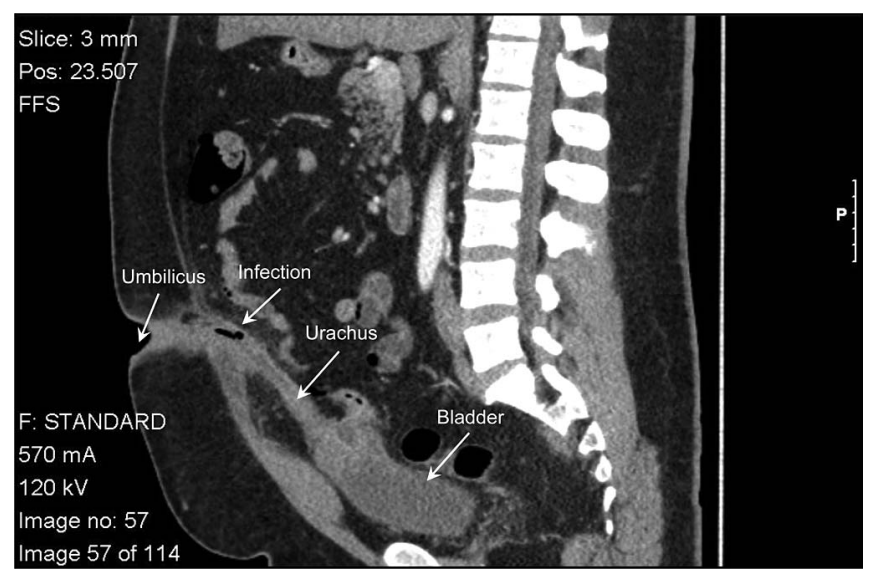

Figure 1. Computed tomographic image of the infected urachal cyst in patient 1 .

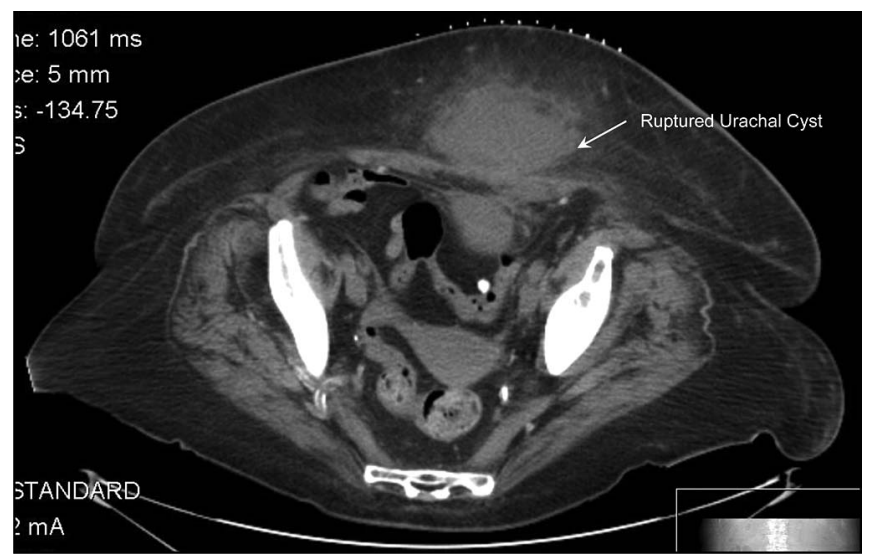

Figure 2. Computed tomographic image of the infected urachal cyst in patient 2 .

11 months later with symptoms of lower abdominal pain, erythema across the lower abdomen, and low-grade fevers. CT imaging revealed a suprapubic phlegmon measuring $5.4 \times 4.8 \mathrm{~cm}$, extending from the bladder dome to the left rectus muscle and umbilicus (Figure 2).

An interventional radiologist performed CT-guided abscess drainage. Infection with methicillin-resistant Staphylococcus aureus (MRSA) was treated with intravenous vancomycin (1500 mg, twice daily) and linezolid (500 mg, twice daily). One week later, open surgical drainage of the abscess was necessary for better control of the infection. CT imaging demonstrated the resolution of the abscess and the presence of a residual urachal lesion.

Six weeks after initial presentation, the patient underwent robotic excision of the urachal lesion with partial cystectomy and closure of the anterior abdominal wound. Pathology was negative for malignancy, and the patient had 
no recurrence of the abscess during 3 years of follow-up; however, she had repeated urinary tract infections that were treated with antibiotics.

\section{DISCUSSION}

Urachal cysts typically arise as a result of desquamation and degeneration of the urachal epithelium, becoming symptomatic if they drain or are infected. ${ }^{13,18}$ During the periumbilical port placement in laparoscopic surgery, the cyst may be inadvertently punctured, leading to the introduction of bacteria and infection or a persistently draining sinus. In rare cases, spontaneous rupture of an infected cyst into the abdominal cavity occurs and can result in sepsis or generalized peritonitis, ${ }^{19}$ which can be severe. ${ }^{11,20,21}$ If the bladder is involved, urinary tract infections and symptoms may also be present. ${ }^{2}$ Although diagnosis is typically made with CT imaging, ultrasonography can also be useful. ${ }^{2}$ In addition, cystoscopy can play a role in determining whether the urachal remnant involves the bladder dome. Draining the abscess may temporarily treat the infection, but recurrence is common, and complete excision of the urachal remnant with partial cystectomy is recommended, to prevent recurrence. Because there is a $30 \%$ reinfection rate, complete excision of the cyst wall is important. ${ }^{22}$ In addition, carcinoma may develop in cases of an unresected or incompletely resected urachal remnant. ${ }^{23,24}$ One of the most extensive reviews of urachal cysts to date reported that urachal masses in 66 of 130 patients (50.8\%) were malignant. ${ }^{4}$ If the urachal remnant is completely excised, we do not believe follow-up imaging is necessary to rule out a recurrence. Surgical treatment options include open, laparoscopic, and robotic techniques. ${ }^{14-17}$

Patients with a quiescent and asymptomatic urachal cyst treated by laparoscopic surgery present a unique challenge, in that the cyst may be ruptured inadvertently and become infected during the placement of the periumbilical port used in the procedure. Whenever available, preoperative images should be reviewed carefully for signs of a urachal remnant. However, although a significant number of adult patients have urachal remnants, the incidence of the complication described in this case study is low. We do not believe that routine imaging is warranted, from both safety and cost perspectives. If a urachal remnant is present, urologic consultation should be considered, and, if possible, laparoscopic port placement should be modified to avoid the remnant. If port placement above rather than below the umbilicus is just as effective for a given surgeon, this option should be considered. Furthermore, if a urachal remnant is identified preoperatively, we recom- mend peritoneal access above the umbilicus or at Palmer's point.

Proper treatment of an infected urachal cyst is critical to the successful recovery of the patient. Investigational imaging before laparoscopic surgery may help to avoid the puncture of an asymptomatic urachal cyst during laparoscopic periumbilical port placement. Our patients underwent laparoscopic cholecystectomy at 11 months and 4 years before presentation. Patient 1 experienced occasional umbilical drainage after the surgery-a likely indication of conversion of a urachal cyst to a urachal sinus. Although it may not be possible to screen all patients before placement of the laparoscopic port, images are often already available and should be reviewed before surgery. Unfortunately, in both of our cases, no useful preoperative images were available. Ultrasonographic images generally do not include the pelvis in patients presenting with upper abdominal complaints.

Our case reports highlight several important concerns relevant to patients who undergo laparoscopic surgery. In both patients, the placement of a periumbilical port during laparoscopic surgery was the likely cause of the puncture and infection of a latent, asymptomatic urachal cyst. Subsequent symptoms of abdominal discomfort did not immediately elicit an accurate diagnosis. If available, review of imaging to identify urachal lesions before periumbilical port placement is recommended, and care should be taken during the placement of laparoscopic periumbilical ports to avoid urachal anomalies if present.

\section{CONCLUSIONS}

Infected urachal cysts can present after laparoscopic port placement. Acute treatment of the infection is necessary. However, long-term control often requires surgical excision. Effective treatment options are available, but prevention of these infections would be ideal. Although it may not be possible to screen all patients before laparoscopic periumbilical port placement for urachal lesions, images are often available and should be reviewed, to avoid complications that arise from a ruptured urachal cyst.

\section{References:}

1. Moore KL. The Developing Human. 2nd ed. Philadelphia: WB Saunders; 1977:113.

2. Laboccetta LT, Picard JC. The urachus: a review and update. AUA Update Series. 2012;31:89-95.

3. Castillo OA, Vitagliano G, Olivares R, Sanchez-Salas R. Complete excision of urachal cyst by laparoscopic means: a new 
approach to an uncommon disorder. Arch Esp Urol. 2007;60: 607-611.

4. Ashley RA, Inman BA, Routh JC, Rohlinger AL, Husmann DA, Kramer SA. Urachal anomalies: a longitudinal study of urachal remnants in children and adults. J Urol. 2007;178:1615-1618.

5. Ozbek SS, Pourbagher MA, Pourbagher A. Urachal remnants in asymptomatic children: gray-scale and color Doppler sonographic findings. J Clin Ultrasound. 2001;29:218-222.

6. Robert YR, Hennequin-Delerue C, Chaillet D, et al. Urachal remnants: sonographic assessment. J Clin Ultrasound. 1996;24: 339-344.

7. Schubert GE, Pavkovic MB, Bethke-Bedurftig. Tubular urachal remnants in adult bladders. J Urol. 1982;127:40-42.

8. Yoo KH, Lee SJ, Chang SG. Treatment of infected urachal cysts. Yonsei Med J. 2006;47:423-427.

9. Ekwueme KC, Parr NJ. Infected urachal cyst in an adult: a case report and review of the literature. Cases J. 2009;25:2:6422-6424.

10. MacNeily AE, Koleilat N, Kiriuluta HG, Homsy YL. Urachal abscesses: protean manifestations, their recognition and management. Urology. 1992;40:530-535.

11. Horgan PG, Johnson S, Courtney D. Intraperitoneal rupture of an infected urachus. BrJ Urol. 1994;73:216.

12. Tazi F, Ahsaini M, Khalouk A, et al. Abscess of urachal remnants presenting with acute abdomen: a case series. J Med Case Rep. 2012;6:226.

13. Yu JS, Kim KW, Lee HJ, Lee YJ, Yoon CS, Kim MJ. Urachal remnant diseases: spectrum of CT and US findings. Radiographics. 2001;21:451-461.

14. Bertozzi M, Riccioni S, Appignani A. Laparoscopic treatment of symptomatic urachal remnants in children. J Endourol. 2014: 28:1091-1096.
15. Sun J, Zhu YJ, Shi CR, et al. Laparoscopic radical excision of urachal remnants with recurrent infection in infants. J Endourol. 2010;24:1329-1332.

16. Okegawa T, Odagane A, Nutahara K, et al. Laparoscopic management of urachal remnants in adulthood. Int J Urol. 2006; 13:1466-1469.

17. Madeb R, Knopf JK, Nicholson C, et al. The use of robotically assisted surgery for treating urachal anomalies. BJU Int. 2006;98: $838-842$.

18. Avni EF, Matos C, Diard F, Schulman CC. Midline omphalovesical anomalies in children: contribution of ultrasound. Urol Radiol. 1998;10:189-194.

19. Agatstein EH, Stabile BE. Peritonitis due to intraperitoneal perforation of infected urachal cysts. Arch Surg. 1984;119:12691273.

20. Iuchtman M, Rahav S, Zer M, Mogliner J, Siplovich L. Management of urachal anomalies in children and adults. Urology. 1993; $42: 426-430$.

21. O'Connor JP, Biyani CS, Austin OM, Browning AJ. Necrotizing fasciitis: a rare complication of an infected urachal remnant in adults. J Urol. 2003;170:920-921.

22. Goldman IL, Caldamone AA, Gauderer M, et al. Infected urachal cysts: a review of 10 cases. J Urol. 1988;140:375-378.

23. Friedland GW, Devries PA, Matilde NM, Cohen R, Rifkin MD. Congenital anomalies of the urinary tract. In: Pollack HM, ed. Clinical Urography. Philadelphia, PA: Saunders, 1990; 559-787.

24. Blichert-Toft M, Nielsen OV. Congenital patent urachus and acquired variants: diagnosis and treatment- review of the literature and report of five cases. Acta Chir Scand. 1971;137:807814 . 